\title{
Latest results from Double Chooz
}

\author{
Inés Gil-Botella*i \\ Centro de Investigaciones Energéticas, Medioambientales y Tecnológicas (CIEMAT) \\ E-mail: ines.gileciemat.es
}

Double Chooz (DC) is a reactor neutrino experiment running at the Chooz nuclear power plant in France. In 2011, DC first reported indication of non-zero $\theta_{13}$ neutrino mixing angle with the far detector (FD) located at the maximum of oscillation effects. A robust observation of $\theta_{13}$ followed in 2012 by other reactor experiments with multiple detector configurations. In the DC single detector configuration, the precision of $\theta_{13}$ was totally dominated by the reactor flux uncertainty. Since 2015 DC runs in a multi-detector configuration reducing strongly the impact of several otherwise dominating systematics. Due to the unique almost iso-flux site configuration, the DC near detector (ND) becomes a direct accurate non-oscillation reference to the FD.

The most recent DC $\theta_{13}$ measurement with two detectors uses a novel analysis method aimed to maximize the accuracy; i.e. the goodness of the central value of $\theta_{13}$. Despite a major increase of statistical power, a conservative approach on systematics has been adopted for now. The latest multi-detector DC $\theta_{13}$ value presents a deviation with respect to the world average. The combined "reactor- $\theta_{13}$ " measurement is expected to remain as the world reference for decades, relying on systematic uncertainties in the per mil level. Thus, the redundancy of multiple experiments is critical to ensure the accuracy and precision of the measurement, which is reference for current and future projects sensitive to $\mathrm{CP}$-violation and atmospheric mass hierarchy observables.

Beyond oscillation physics, DC has articulated one of the most precise single-detector setups, allowing high precision reactor spectrum characterization, including both shape and normalization. This is a particularly hot topic since DC provided the first evidence of a sizeable reactor neutrino spectral distortion in May 2014, confirmed later on by other experiments, questioning the accuracy and precision of today's reactor neutrino predictions technology.

The European Physical Society Conference on High Energy Physics

5-12 July, 2017

Venice

\footnotetext{
* Speaker.

$\dagger$ on behalf of the Double Chooz collaboration.
} 


\section{The Double Chooz detectors}

Double Chooz (DC) is a reactor neutrino experiment aiming to provide a precise measurement of the $\theta_{13}$ mixing angle. The antineutrinos used in DC are produced by the pair of reactors (type N4) located at the CHOOZ-B nuclear power station in France. The maximum operating thermal power of each core amounts to $4.27 \mathrm{GW}$. DC has two almost identical neutrino detectors of medium size, containing 10.3 cubic meters of liquid scintillator target doped with $1 \mathrm{~g} / \mathrm{l}$ of gadolinium. The DC far detector is located at $1.05 \mathrm{~km}$ from the two reactor cores and shielded by about 300 mwe of rocks. In order to cancel the systematic errors originated from the nuclear reactors (lack of knowledge of the $\bar{v}_{e}$ flux and spectrum) as well as to reduce the set of systematic errors related to the detector and event selection procedure, a second detector is installed close to the nuclear cores, at $\sim 400$ $\mathrm{m}$. At such a rather simple site configuration with just two reactors and two detectors at almost iso-flux positions, the flux related uncertainty becomes negligible compared to other uncertainties as efficiency and backgrounds.

The reactor antineutrinos are detected through the inverse beta decay reaction where neutrinos interact with protons giving a prompt signal due to the $\mathrm{e}^{+}$scintillation and annihilation and a delayed signal from the neutron capture on a certain nuclei. The prompt signal is the $\mathrm{e}^{+}$kinetic energy and the gammas from $\mathrm{e}^{+}$annihilation. Measuring the $\mathrm{e}^{+}$energy, we can obtain the neutrino energy. The energy threshold of this reaction is $1.8 \mathrm{MeV}$. The delayed signal corresponds to the neutron which is thermalized and then captured by gadolinium or hydrogen. In the case of $\mathrm{H}$, it happens $\sim 200 \mu$ s after the $\mathrm{e}^{+}$annihilation and it gives gammas of $2.2 \mathrm{MeV}$. In the case of $\mathrm{Gd}$, the time delay is $\sim 30 \mu$ s giving gammas of $8 \mathrm{MeV}$. This characteristic signature yields a very efficient background rejection.

\section{Single-detector analyses}

DC started the data taking with the far detector in April 2011 and presented its first results in November 2011 with $15.34 \mathrm{GW}$-ton-years of exposure [1]. DC provided the first indication of a non-zero value of $\theta_{13}$ by a reactor-based experiment $\left(\sin ^{2} 2 \theta_{13}=0.086 \pm 0.041\right)$. Since then, many efforts and advances have been performed by the collaboration to improve this result, using the data with the FD while the ND was under construction. Updated analyses on $\theta_{13}$ were published in 2012 [2] and 2014 [3] with additional data and a better energy reconstruction and additional muon vetos. Additionally, in the last analysis, several novel techniques have been developed to achieve significant reductions of the backgrounds and systematic uncertainties with respect to previous publications, whereas the efficiency of the signal has increased. The value of $\theta_{13}$ is measured to be $\sin ^{2} 2 \theta_{13}=0.090+0.032-0.029$ from a fit to the observed energy spectrum.

A second independent measurement of $\theta_{13}$ has been performed by DC using a different analysis technique: the reactor rate modulation (RRM) analysis [4]. The rate of neutrino candidates is measured during periods of different reactor power ranging from zero power (reactor-off) to full power. This analysis allows the measurement of $\theta_{13}$ without an a priori knowledge of the background. The RRM method is unique to DC because of its simpler site, which includes only two reactors. DC also measured its irreducible backgrounds in situ by means of seven days of reactoroff data confirming the reliability of the background model used in the oscillation analysis [5]. 
A new analysis based on neutron captures on hydrogen was published [6] providing for the first time a measurement of $\theta_{13}$ using this technique. Given the fact that $\mathrm{H}$ and $\mathrm{Gd}$ neutrino candidates are disjoint samples, their corresponding $\theta_{13}$ measurements have been combined, considering all correlated terms such as the flux, response and detection systematics, to obtain a more precise measurement of $\theta_{13}$ [7]. An unexpected spectrum distortion is observed at high energy (4-6 MeV) but its impact on the $\theta_{13}$ measurement is evaluated to be insignificant with respect to the uncertainty. A strong correlation between the excess rate and the reactor power is observed, excluding the possibility to an unknown background.

\section{Results with two-detector data}

Since Dec 2014, DC is taking data with both near and far detectors simultaneously. After nine months of data with two detectors, DC provided the first multi-detector $\theta_{13}$ measurement $\left(\sin ^{2} 2 \theta_{13}\right.$ $=0.111 \pm 0.018$ ) with a central value slightly higher than the one from Daya Bay [8] (about 1.6 $\sigma$ difference). Thanks to the simple setup with 2 reactors and 2 detectors, DC can almost completely cancel the uncertainty from the reactor flux. All systematics uncertainties are below $0.4 \%$ in the rate and shape analysis. The precision of the measurement was limited by the low statistics.

With the aim of increasing the statistical power of the experiment, a new analysis was developed by including neutron captures on hydrogen in the neutrino selection ( $\mathrm{Gd}+\mathrm{H}$ analysis). The idea of the new analysis is to increase the neutrino target by including the Gamma Catcher (GC) volume, which has liquid scintillator without Gd and include all neutron captures inclusively.

The previous Gd analysis just looked at neutrino interactions with a delay signal from the neutron capture on $\mathrm{Gd}$ at $\sim 8 \mathrm{MeV}$ where the accidental background was low. A new technique of analysis using an Artificial Neural Network (ANN) has been developed to properly reject the accidental background in the $2.2 \mathrm{MeV} \mathrm{H}$-signal region. The ANN is based on three variables which are different from signal and accidental background: the delayed energy, the correlation time and the correlation distance. Fig. 1 shows how the accidental background present in the delay energy distribution is reduced by applying a cut in the ANN variable.
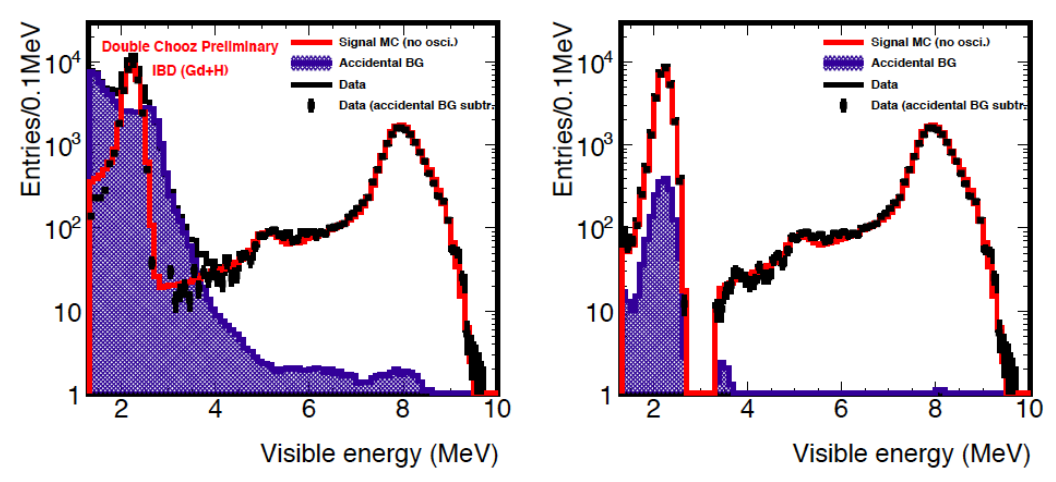

Figure 1: Delay energy spectrum before (left) and after (right) ANN selection was applied.

The neutrino rate in the near (far) detector is increasing to about 900 (140) events per day. It was demonstrated that the background level could be kept under control and the signal to background ratio in both detectors is still well above 10 with the new selection. 
Three different sets of data, FD-I, FD-II and ND, are used for the recent results of DC. FD-I and FD-II are FD data taken before and after ND started to work, respectively. $\theta_{13}$ is measured by the data to prediction simultaneous fit on the event rate and the prompt energy of each dataset. The prediction is constructed with signal Monte Carlo and backgrounds estimated from data. Reactor off data is used to constrain background. Systematic uncertainty correlations between datasets are considered in the fit.

Fig. 2 shows the ratio of the observed energy spectrum to the non oscillation prediction for FD-I, FD-II and ND. The best fit value is $\sin ^{2} 2 \theta_{13}=0.119 \pm 0.016$ and $\chi^{2} / \mathrm{ndf}=236.2 / 114$.
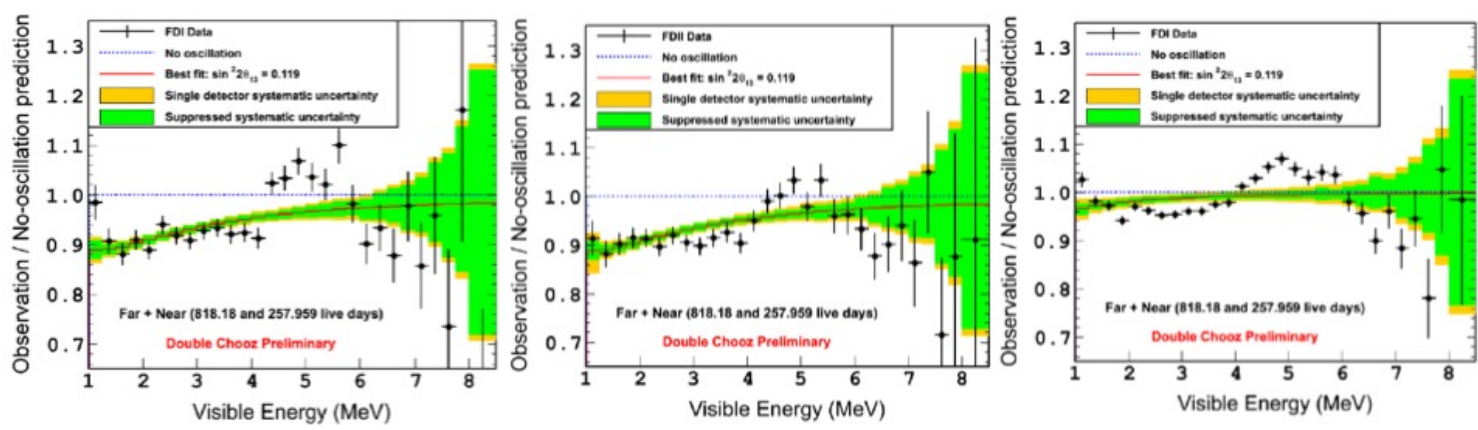

Figure 2: Ratio of the observed energy spectrum to the prediction for all data sets (FD-I, FD-II and ND). Red lines show the best-fit value. Systematics uncertainties are also plotted.

There are some structures in the prompt energy neutrino spectrum between 4 and $6 \mathrm{MeV}$ not expected from shape calculations. This spectral distortion is also visible in other experiments [9] and it is under study. In order to cross check the previous $\theta_{13}$ results, another fit is performed using the same period of data of FD-II and ND. Fig. 3 shows the ratio of FD-II to ND and the best fit value is $\sin ^{2} 2 \theta_{13}=0.123 \pm 0.023$, being in good agreement with the data to MC fit within uncertainties.

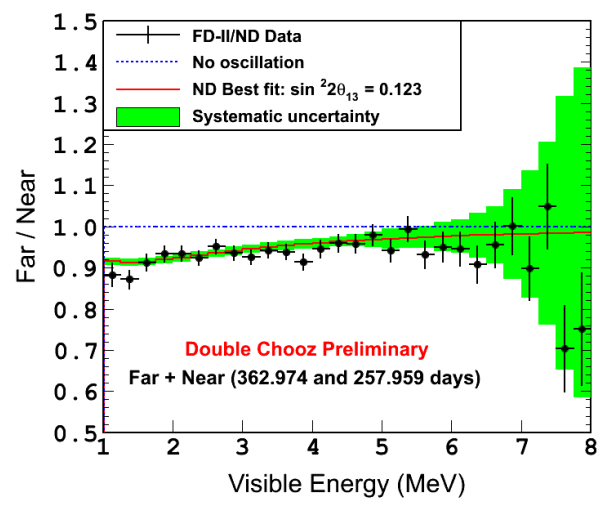

Figure 3: Ratio of the observed energy spectrum from FD-II to that from the ND data after the correction for the different baselines. The red line shows the best-fit value. Systematics uncertainties are also plotted. 


\section{Prospects}

The latest DC results are not anymore dominated by the reactor flux thanks to the ND data. The development of the new $\mathrm{Gd}+\mathrm{H}$ analysis also allowed the reduction of the statistical uncertainty. The current largest systematic uncertainty is the detector uncertainty, which is dominated by the proton number uncertainty. Fig. 4 shows the evolution of the sensitivity as a function of data taking time in the case the measurement of the proton number can be improved after the detector dismantling. A total uncertainty on $\sin ^{2} 2 \theta_{13}$ below 0.01 could be achieved.

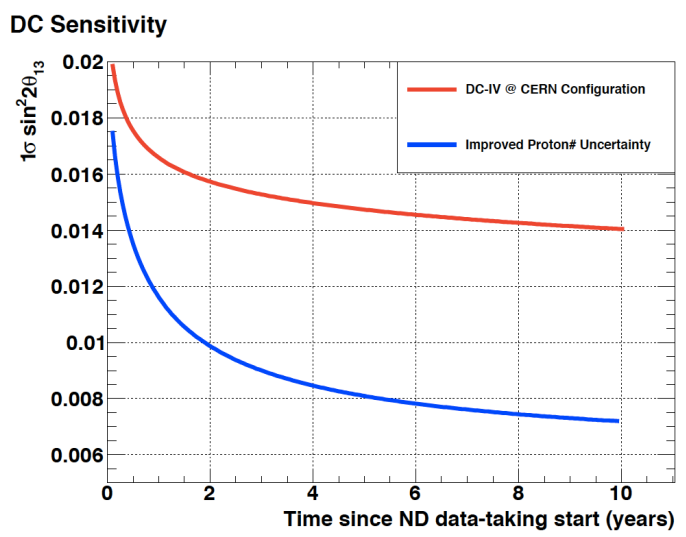

Figure 4: Double Chooz $\sin ^{2} 2 \theta_{13}$ sensitivity evolution including the prospect for an improved proton number estimation.

\section{References}

[1] Y. Abe et al., [Double Chooz Collaboration], Phys. Rev. Lett. 108 (2012) 131801

[2] Y. Abe et al. [Double Chooz Collaboration], Phys. Rev. D 86 (2012) 052008

[3] Y. Abe et al. [Double Chooz Collaboration], JHEP 1410 (2014) 086; Erratum: [JHEP 1502 (2015) 074]

[4] Y. Abe et al. [Double Chooz Collaboration], Phys. Lett. B 735 (2014) 51

[5] Y. Abe et al. [Double Chooz Collaboration], Phys. Rev. D 87 (2013) no.1, 011102

[6] Y. Abe et al. [Double Chooz Collaboration], Phys. Lett. B 723 (2013) 66

[7] Y. Abe et al. [Double Chooz Collaboration], JHEP 1601 (2016) 163

[8] F. P. An et al. [Daya Bay Collaboration], Phys. Rev. D 95 (2017) no.7, 072006

[9] F. P. An et al. [Daya Bay Collaboration], Phys. Rev. Lett. 116 (2016) no.6, 061801 Erratum: [Phys. Rev. Lett. 118 (2017) no.9, 099902]

S. H. Seo et al. [RENO Collaboration], arXiv:1610.04326 [hep-ex]

Y. J. Ko et al., Phys. Rev. Lett. 118 (2017) no.12, 121802 